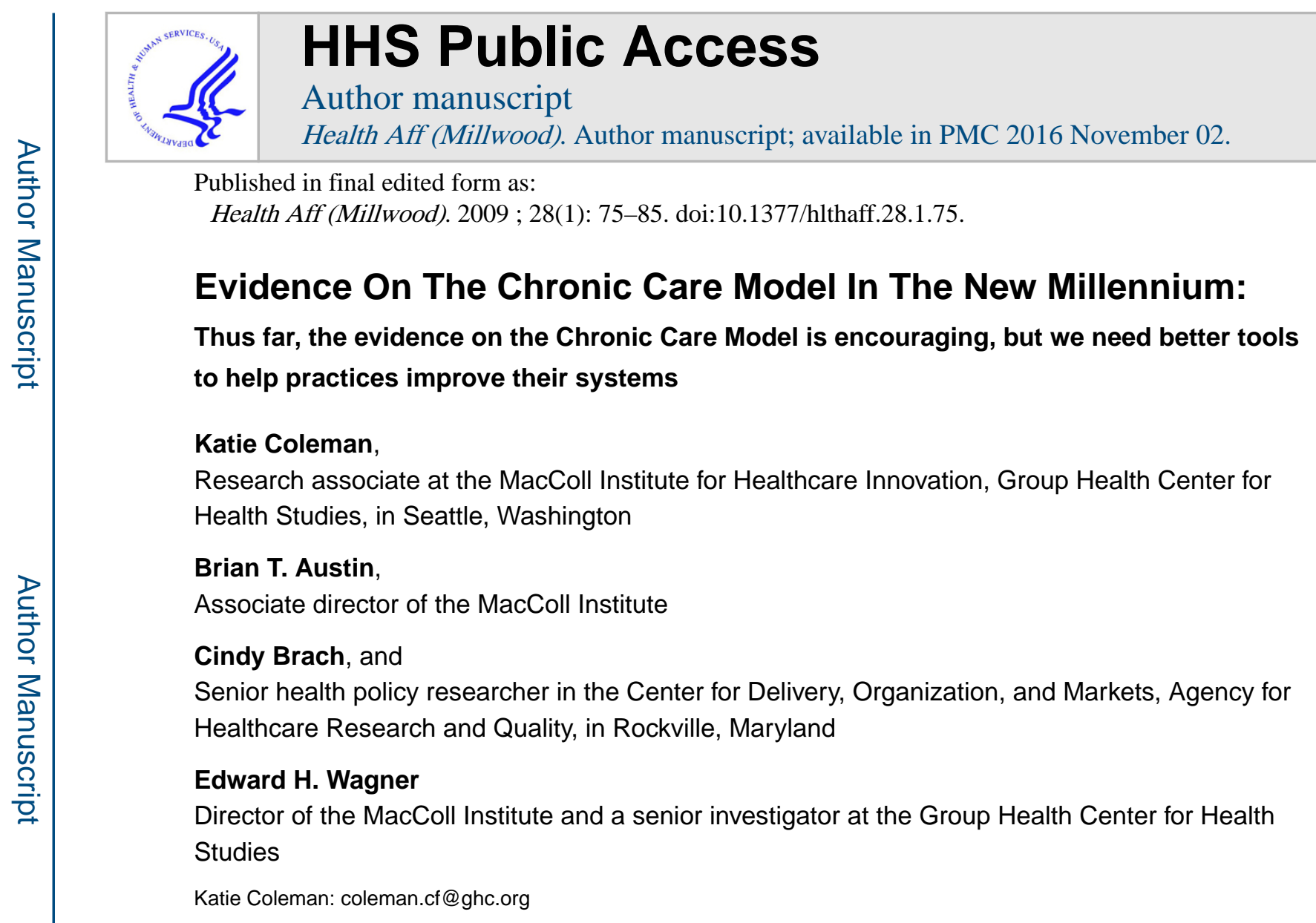

\title{
Abstract
}

Developed more than a decade ago, the Chronic Care Model (CCM) is a widely adopted approach to improving ambulatory care that has guided clinical quality initiatives in the United States and around the world. We examine the evidence of the CCM's effectiveness by reviewing articles published since 2000 that used one of five key CCM papers as a reference. Accumulated evidence appears to support the CCM as an integrated framework to guide practice redesign. Although work remains to be done in areas such as cost-effectiveness, these studies suggest that redesigning care using the CCM leads to improved patient care and better health outcomes.

Chronic diseases are now the major cause of death and disability worldwide, responsible for 59 percent of deaths and 46 percent of the global burden of disease. ${ }^{1}$ Despite advances in the effectiveness of treatment, research shows that patients frequently do not get the care they want or need. ${ }^{2}$ The Chronic Care Model (CCM) is designed to help practices improve patient health outcomes by changing the routine delivery of ambulatory care through six interrelated system changes meant to make patient-centered, evidence-based care easier to accomplish. The aim of the CCM is to transform the daily care for patients with chronic illnesses from acute and reactive to proactive, planned, and population-based. It is designed to accomplish these goals through a combination of effective team care and planned interactions; self-management support bolstered by more effective use of community resources; integrated decision support; and patient registries and other supportive information technology (IT). These elements are designed to work together to strengthen the provider-patient relationship and improve health outcomes. 
In this paper we review evidence published since 2000 about practices' ability to redesign care in accord with the CCM and the impacts of such redesign on clinical care and health outcomes. We then discuss implications for practice and research.

\section{A Brief History Of The CCM}

The initial evidence upon which the CCM was based came from a review of interventions to improve care for various chronically ill populations. ${ }^{3}$ These evaluations showed-and a subsequent Cochrane Collaboration review confirmed - that multicomponent practice changes in four categories led to the greatest improvements in health outcomes: increasing providers' expertise and skill, educating and supporting patients, making care delivery more team-based and planned, and making better use of registry-based information systems. ${ }^{4}$ These changes formed the basis of the CCM. ${ }^{5}$

Today the CCM is a widely adopted approach to ambulatory care improvement. It guides national quality improvement initiatives involving groups of primary care practices, such as the Health Disparities Collaboratives (HDCs), as well as state-based and regional efforts that, combined, have worked with more than 1,500 physician practices in the United States and internationally. The CCM is also an integral part of current patient-centered medical home models. ${ }^{6}$

Until recently, evidence supporting the CCM was largely inferred from studies of its individual components. Over the past ten years, evaluations, trials, and organizational effectiveness studies have examined whether changing ambulatory care through the integrated set of practice changes that constitute the CCM can improve patient care and lead to better health.

\section{Study Methods}

For this work, a CCM-based intervention was defined as different from disease management or other interventions to improve the care of complex chronic illnesses. To be included in this study, an intervention had to operate within ambulatory care practices, changing how daily care is delivered by clinical teams. ${ }^{7}$ To be defined as a CCM, an intervention had to require clinical teams to work differently, not just refer patients to external support. Second, it had to be multicomponent. To be defined as CCM-based, an intervention had to integrate changes that involved most or all of the six areas of the model: self-management support, decision support, delivery system design, clinical information systems, health care organization, and community resources.

\section{Literature search}

Variations in nomenclature used by authors and imprecise descriptions of interventions made it difficult to meaningfully identify CCM-based interventions using PubMed. Therefore, to facilitate the search for and collection of relevant articles, we used the Science Citation Index Web of Knowledge search tool to gather articles published between January 2000 and March 2008 that cite one of five articles that together originally described the CCM. ${ }^{8}$ We also used bibliographic searches and expert consultation to find additional articles. Limiting 
the search to articles and reviews in English identified a universe of 944 U.S. and international papers.

\section{Criteria for inclusion}

For an article to be included in our final analysis, it had to be an empirical evaluation of a CCM-based intervention or an observational study examining the relationship between the presence of CCM elements and health or financial outcomes. Specifically, a study was deemed to be based on the CCM if the authors described their intervention as based on the $\mathrm{CCM}$ and if the intervention attempted to make changes in four or more elements of the model together to redesign ambulatory care. We rejected any interventions that occurred outside of the primary care practice, including, for example, case management programs operated by external disease management companies as well as single-component interventions. In addition, we included only studies with community-based patients who were identified and treated in primary care. Specialty-based clinic programs, rehabilitation programs, and telehealth programs focused on providing home care or testing a specific device were excluded.

The articles were reviewed by one of three researchers to find interventions that met the above criteria. Questions about inclusion arose: can changes in four or more elements of the $\mathrm{CCM}$ be deduced from the intervention description, even if not explicitly mentioned by the authors as discrete system changes? Or, how closely integrated with primary care does an intervention need to be for it to qualify as "aimed at improving care delivery"? These types of questions were resolved through group discussion among the three reviewers.

\section{Retained bibliography}

Eighty-two articles were retained for the final study: fourteen reviews or meta-analyses; twenty-one studies of the relationship between organizational characteristics and quality improvement; eleven articles describing nine randomized control trials of CCM-based interventions; six studies related to cost or cost-effectiveness; and thirty quasi-experimental or observational evaluations of the use of the CCM in quality improvement. Most articles reflect experience in the United States, although European, Canadian, and Australian experience is represented as well. We then used these articles to answer several research questions. ${ }^{9}$

\section{Does The CCM Improve The Delivery Of Care And Patient Health Outcomes?}

Over the past decade, many health care organizations have used the CCM to guide chronic care improvement either through participation in a Breakthrough Series (BTS) Collaborative or on their own. ${ }^{10}$ The largest concentration of evidence emanates from evaluations of practices that learned about the CCM by participating in a BTS collaborative because the collaboratives have been, and continue to be, the dominant method used to help practices implement the CCM. Two major quality improvement efforts-the Health Resources and Services Administration's (HRSA's) Health Disparities Collaboratives (HDCs) and the 
Improving Chronic Illness Care (ICIC) Collaboratives-provide information about the realworld challenges of implementing and sustaining the CCM in busy practices.

\section{Implementing the CCM}

RAND examined fifty-one organizations participating in four collaboratives. ${ }^{11}$ Two questions posed by their evaluation were as follows: Can busy practices implement the CCM? And, if they can, will their patients benefit? Intervention practices were able to implement the CCM, making an average of forty-eight practice changes across all six CCM elements. ${ }^{12}$ Three-fourths of practices sustained these changes one year later, and about the same proportion spread the CCM to new sites or conditions.

\section{Evidence of improved care}

Patients of intervention practices received improved care. Compared to patients in control practices, patients of providers actively participating in the congestive heart failure (CHF) collaborative were more knowledgeable, used recommended therapies such as lipid-lowering and angiotensin-converting enzyme (ACE) inhibition therapy more often, visited the emergency department (ED) less often, and experienced 35 percent fewer days in the hospital. ${ }^{13}$ When their practices redesigned care according to the CCM, patients with asthma were more likely than patients whose practices did not redesign care to monitor their peak flows and have a written action plan, and their quality of life improved. ${ }^{14}$ Also, diabetic patients experienced reduced risk of cardiovascular disease; for every forty-eight patients who received care from a redesigned practice, risk declined by one cardiovascular disease event. ${ }^{15}$

\section{Negative results}

However, Charles Homer and colleagues did not find such results in their randomized trial of collaborative participation. In their study, forty-three pediatric practices interested in improving asthma care were assigned into either an intervention group or a wait-list control group. Interviews with parents of asthmatic patients before and immediately after the collaborative revealed no differences between groups in either processes or outcomes of care. ${ }^{16}$ The short follow-up period, low participation rates, and possible contamination between the intervention and control practices that were part of the same organization were among the reasons given by the authors for the negative results.

\section{HRSA's collaboratives}

Three recently published large evaluations of HRSA's HDCs provide valuable information about the effectiveness of the CCM and the time it may take to discern changes in intermediate health outcomes.

Marshall Chin and colleagues studied nineteen midwestern community health centers (CHCs) involved in diabetes collaboratives. ${ }^{17}$ Unlike Homer and colleagues, who examined parents' self-reports, Chin and colleagues reviewed the medical records of a random sample of each CHC's diabetic patients in the years before and during the collaborative. They found significant improvements in the processes of care, but not in intermediate outcomes. 
Bruce Landon and colleagues studied a similar sample of CHCs that participated in the HDCs. Asthma and diabetes patients in participating CHCs showed significantly greater improvements in process measures such as use of anti-inflammatory medication for asthma and foot screening for diabetes in the year after the completion of the collaborative than did patients in control CHCs. They, like Chin and colleagues, found no differences in intermediate outcomes such as hemoglobin A1c or blood pressure levels. ${ }^{18}$ These two evaluations raised many questions, including whether process improvements would lead to outcome improvements and whether a one-year follow-up period was long enough to detect changes in health outcomes.

To address these questions, Chin and colleagues expanded the average time patients were followed after the intervention, from one year to three years, in a second evaluation of thirtyfour HDC-participating centers. ${ }^{19}$ They confirmed their earlier finding that only process improvements were detected immediately after the HDC. However, two years later they noted significant improvements in intermediate outcomes such as HbA1c and low-density lipoprotein (LDL) levels.

These three HDC evaluations considered patients from anywhere in the $\mathrm{CHC}$ to be part of the intervention, not just the patients of those practices actively engaged in quality improvement, as in the RAND evaluation. In so doing, they obtained findings that bolstered emerging evidence that the collaborative learning structure and the CCM were effective models for improving care processes, although teams may have to wait to see real improvements in clinical outcomes. They also showed that teams were able to sustain the improvements and spread them across their organization.

\section{Other evaluations}

Many other quality improvement evaluations based on the CCM have been published. Most relied on the data provided by the participating teams rather than more objective, externally collected evaluation data. All showed improvement on some process measures, and most also showed improvement on some intermediate outcome measures such as HbA1c, LDL cholesterol, and arterial pressure. ${ }^{20}$ Because of limitations in the study designs of these evaluations, we cannot conclude that these changes resulted solely from CCM-guided efforts.

\section{Results from randomized trials}

However, the results are bolstered by the findings of randomized trials of noncollaborative CCM interventions that show similar results. Unlike the BTS collaborative interventions described above, these research-based interventions generally involved the addition of new staff and other resources, which might facilitate implementation and limit the extent of internal practice redesign. All trials found that implementation of the CCM significantly improved at least some process and outcome measures compared to controls across a variety of diseases, including comorbid depression and cancer. ${ }^{21}$ 


\section{Is A Complex, Multicomponent Model Really Necessary?}

Recent randomized controlled trials, meta-analyses, and observational studies address the importance of an integrated approach to improving chronic illness care. ${ }^{22}$ The CCM was designed to build on the interrelationships between six evidence-based elements that lead to improved clinical quality. For example, for patients to engage in proactive care (delivery system design), practices need to be able to view all of the patients in their panels (clinical information systems) who need certain guideline-based treatments (decision support), and patients must agree to any changes in their care and integrate them into their lives (selfmanagement support). As such, CCM-based interventions focus on practice redesign across most or all of the six elements. However, RAND's evaluation shows that practice teams implement some of the elements with more ease and in greater depth than others; information systems received the most attention, and community linkages, the least. ${ }^{23}$

\section{Results of using only some CCM elements}

Several observational studies have examined the relationship between the presence of CCM elements and quality of care. In general, all studies found that composite measures of CCM implementation were significantly associated with either improvements in or higher levels of quality of care as measured by process or outcome measures. For example, Michael Parchman and colleagues found that HbA1c scores and ten-year risk of heart disease were lowest in diabetic patients whose primary care conformed most to the CCM. ${ }^{24}$

In addition, some of these studies attempted to identify either individual CCM elements or specific interventions included in CCM elements that accounted for the positive effect. These studies found that the presence of multiple CCM elements were associated with better quality of care. For example, Barbara Fleming and colleagues used an organizational assessment tool based on the CCM to compare high-performing (top quartile) and lowperforming (bottom quartile) Medicare Advantage (MA) organizations. They found that organizations with higher patient satisfaction and stronger diabetes quality outcomes (high performers) were more likely than their peers to organize care delivery in accordance with the CCM. High-performing organizations more often used computerized reminders (clinical information systems), involvement of practitioners on quality improvement teams (health care organization), guidelines supported by clinician education or computer support (decision support), formal self-management programs (self-management support), and a registry (clinical information systems). ${ }^{25}$ Damin Si and colleagues studied Aboriginal health centers in Australia. They found that four of the six CCM elements (health care organization, community linkages, delivery system design, and clinical information systems) were independently associated with the quality of diabetes care. ${ }^{26}$ Jo Ann Sperl-Hillen and colleagues found that only delivery system design was significantly correlated with better diabetes outcomes in clinics associated with a large health system, but two others, selfmanagement support and clinical information systems, "demonstrated associations that may have substantive significance." ${ }^{27}$ Researchers acknowledge the challenges of disentangling multicomponent interventions. ${ }^{28}$ 


\section{Is The CCM Cost-Effective?}

There is some evidence that interventions that result in improved disease control reduce total health care costs for patients with CHF and for diabetics with higher HbA1c scores. ${ }^{29}$ Todd Gilmer and colleagues confirmed that interventions that focus on clinical meetings and registries for diabetes or heart disease care are associated with lower future costs. ${ }^{30}$

However, cost savings resulting from improved disease control take time to materialize and often accrue to insurers rather than to ambulatory care practices. The experiences of early collaborative participants demonstrated that redesigning practices along the lines of the CCM costs practices money in the short term: an extra \$6-\$22 per patient in the first year, according to one estimate. ${ }^{31}$ Some of these expenses may be attributable to implementation glitches, such as practices' creating redundant workarounds or not capturing reimbursement for group visits. Despite the potential mismatch between who bears the costs to implement the CCM and who potentially receives the financial benefits, Elbert Huang and colleagues found that reduced risk of blindness, end-stage renal disease, and coronary artery disease resulted in an increase in quality-adjusted life-years (QALYs) at a price considered to be cost-effective from a societal perspective. ${ }^{32}$ Evidence on the cost-effectiveness of the CCM is just beginning to emerge, and more research is needed to understand the costs and benefits to practices, payers, and patients.

\section{Discussion}

Considerable experience using the CCM to improve the quality of chronic illness care has accumulated over the past decade. Although not definitive, published evidence suggests that practices redesigned in accord with the CCM generally improve the quality of care and the outcomes for patients with various chronic illnesses. This finding appears to be consistent in both U.S. and international settings. Although simpler interventions would be attractive, the observational studies reviewed suggest that high-performing practices make changes across multiple elements of the CCM. But much more needs to be learned about the practicality, effectiveness, and cost implications of changing the organization and functioning of ambulatory care in this way. Most of the studies cited here involve highly motivated practices focusing on patients with a single chronic condition. Only limited evidence to date provides assurance that the practice changes become sustained and spread to the care of other illnesses or to other less motivated practices in an organization.

In addition, the CCM is not a discrete, immediately replicable intervention; it is a framework within which care delivery organizations translate general ideas for change into specific, often locally distinctive applications. As a result, the specific practice changes associated with a particular CCM element vary from organization to organization and from country to country. For example, some practices may provide self-management support by referring patients to hospital- or community-based nurse educators, while other practices use office staff trained in motivational interviewing or other counseling methods. Whether or not particular ways of implementing the components of the CCM influence the likelihood of improvement in outcomes requires further research. Such research would be improved through the use of a standardized categorization of the changes made. 


\section{Influence of practice characteristics}

In CCM-based quality improvement efforts, considerable variation in the degree of health outcome improvement among participating organizations is the norm. Although the variation may be correlated with the nature or extent of CCM implementation, contextual factors within each practice setting may also influence the ability of teams to make changes or improve care. Many types of practices have used the CCM to redesign their care. But most of the published experience with the CCM to date pertains to larger practice organizations with clinical IT and other resources. It seems likely that smaller practices, or those with limited IT or nonphysician clinical staff, would have greater difficulty implementing the $\mathrm{CCM}$ and improving outcomes; however, further research is needed to clarify the practice characteristics predictive of success. Further research is also needed to understand better the interrelationships between the baseline characteristics of practice organizations, the changes they make with CCM implementation, and changes in care and patient outcomes.

\section{Impact on costs}

Health care organizations must expend considerable resources and effort to transform their practices in accord with the CCM. Although the evidence to date suggests that such transformation can lead to improved patient care and outcomes, the impact on health care costs and revenues remains uncertain and probably varies by condition. Further, the CCM recommends services and modes of delivery that are generally poorly reimbursed or not reimbursed at all in most fee-for-service schemes. The combination of the effort required by busy practices, unsupportive reimbursement, and an uncertain business case have limited widespread implementation of the CCM except by very large organizations such as the Bureau of Primary Health Care. Instead, many Medicaid agencies, health plans, and employers have turned to direct patient disease management in an effort to improve the care of the chronically ill and reduce its costs. Recent evidence and reviews have questioned the effectiveness of disease management programs that are not closely connected to patients' primary care clinicians. ${ }^{33}$

\section{External support for practice transformation}

The U.S. experience to date suggests that some type of external financial incentive and quality improvement support may be essential for widespread practice change, especially for small practices. Regional improvement programs in North Carolina, Minnesota, and Pennsylvania have embarked on programs of broad-scale practice transformation combined with more supportive practice payment strategies. Multistakeholder regional efforts of this sort have the potential to extend and enhance the ad hoc, time-limited projects that have characterized many CCM implementations up to this point and create long-term, sustainable endeavors.

\section{Study limitations}

To identify relevant, empirical studies for this review, we started with all articles that cited any one of five papers describing the origins, development, and early evaluation of the CCM. We chose this strategy to facilitate the selection of chronic disease improvement efforts or disease management programs really modeled on the CCM. However, if articles describing 
interventions consistent with the CCM do not cite these articles, the results here may be incomplete or biased. To explore if a bias toward positive findings was present in our search strategy, we reviewed the articles citing Homer and colleagues' 2005 negative trial of asthma care to see if negative studies missed by our search strategy surfaced. Three of the twenty articles that cited Homer and colleagues are evaluations of interventions that met our definition of being CCM-based. Two of those three presented equivocal results, and one presented positive results. All were identified by our original search strategy.

In the next decade, the impacts of chronic illness on health and health care costs demand that we move beyond the vanguard physician practices that have volunteered for improvement efforts and reach out to the majority of providers. Doing so will require stronger evidence, better tools, and more effective dissemination models for helping practices improve their systems. ${ }^{34}$ Alternatives to the time-intensive learning collaborative model such as coaching and Web-based learning networks are just now being developed and tested in a variety of settings. ${ }^{35}$ The evidence examined here suggests that the CCM should continue to inform systematic efforts to improve care and that those efforts should be rigorously evaluated.

\section{Acknowledgments}

This work was funded by the Robert Wood Johnson Foundation Improving Chronic Illness Care: Transitional Support for Field Building Grant no. 053022 and Improving Chronic Illness Care: Scientific Support Grant no. 58194. This work was also supported in part by a task order from the Agency for Healthcare Research and Quality (AHRQ) titled "Integrating Chronic Care and Business Strategies in the Safety Net." The views expressed in this paper are those of the authors and do not necessarily reflect the official position of AHRQ or the U.S. Department of Health and Human Services. The authors thank the Robert Wood Johnson Foundation and their colleagues at the MacColl Institute for Healthcare Innovation, Group Health Center for Health Studies, for their ongoing support of this work.

\section{NOTES}

1. World Health Organization. Global Strategy on Diet, Physical Activity, and Health. 2003. http:// www.who.int/dietphysicalactivity/publications/facts/chronic/en/index.html (accessed 24 April 2008)

2. McGlynn EA, et al. The Quality of Health Care Delivered to Adults in the United States. New England Journal of Medicine. 2003; 348(26):2635-2645. [PubMed: 12826639]

3. Wagner EH, Austin BT, Von Korff M. Organizing Care for Patients with Chronic Illness. Milbank Quarterly. 1996; 74(4):511-544. [PubMed: 8941260] Wagner EH. Chronic Disease Management: What Will It Take to Improve Care for Chronic Illness? Effective Clinical Practice. 1998; 1(1):2-4. [PubMed: 10345255]

4. Renders CM, et al. Interventions to Improve the Management of Diabetes Mellitus in Primary Care, Outpatient, and Community Settings. Cochrane Database of Systematic Reviews. 2002;

(1):CD001481.

5. Elements of the CCM are described more fully in online Appendix 1 at http:// content.healthaffairs.org/cgi/content/full/28/1/75/DC1.

6. Berenson RA, et al. A House Is Not a Home: Keeping Patients at the Center of Practice Redesign. Health Affairs. 2008; 27(5):1219-1230. [PubMed: 18780904] Iglehart JK. No Place Like HomeTesting a New Model of Care Delivery. New England Journal of Medicine. 2008; 359(12):12001202. [PubMed: 18799555] National Committee for Quality Assurance. Physician Practice Connections-Patient-Centered Medical Home. 2008. http://ncqa.org/tabid/631/default.aspx accessed 11 July 2008

7. Geyman JP. Disease Management: Panacea, Another False Hope, or Something in Between? Annals of Family Medicine. 2007; 5(3):257-260. [PubMed: 17548854] Casalino LP. Disease Management and the Organization of Physician Practice. Journal of the American Medical Association. 2005; 293(4):485-488. [PubMed: 15671434] 
8. Bodenheimer T, Wagner EH, Grumbach K. Improving Primary Care for Patients with Chronic Illness. Journal of the American Medical Association. 2002; 288(14):1775-1779. [PubMed: 12365965] Bodenheimer T, Wagner EH, Grumbach K. Improving Primary Care for Patients with Chronic Illness: The Chronic Care Model, Part 2. Journal of the American Medical Association. 2002; 288(15):1909-1914. [PubMed: 12377092] Wagner. Chronic Disease Management. Wagner EH, et al. Improving Chronic Illness Care: Translating Evidence into Action. Health Affairs. 2001; 20(6):64-78. [PubMed: 11816692] Wagner, et al. Organizing Care.

9. The articles included in each category are listed in online Appendix 2 (Extended Bibliography), as in Note 5.

10. Wagner, et al. Improving Chronic Illness Care.

11. Cretin S, Shortell SM, Keeler EB. An Evaluation of Collaborative Interventions to Improve Chronic Illness Care: Framework and Study Design. Evaluation Review. 2004; 28(1):28-51. [PubMed: 14750290]

12. Pearson ML, et al. Assessing the Implementation of the Chronic Care Model in Quality Improvement Collaboratives. Health Services Research. 2005; 40(4):978-996. [PubMed: 16033488]

13. Asch SM, et al. Does the Collaborative Model Improve Care for Chronic Heart Failure? Medical Care. 2005; 43(7):667-675. [PubMed: 15970781]

14. Mangione-Smith R, et al. Measuring the Effectiveness of a Collaborative for Quality Improvement in Pediatric Asthma Care: Does Implementing the Chronic Care Model Improve Processes and Outcomes of Care? Ambulatory Pediatrics. 2005; 5(2):75-82. [PubMed: 15780018]

15. Vargas RB, et al. Can a Chronic Care Model Collaborative Reduce Heart Disease Risk in Patients with Diabetes? Journal of General Internal Medicine. 2007; 22(2):215-222. [PubMed: 17356989]

16. Homer CJ, et al. Impact of a Quality Improvement Program on Care and Outcomes for Children with Asthma. Archives of Pediatrics and Adolescent Medicine. 2005; 159(5):464-469. [PubMed: $15867121]$

17. Chin MH, et al. Improving Diabetes Care in Midwest Community Health Centers with the Health Disparities Collaborative. Diabetes Care. 2004; 27(1):2-8. [PubMed: 14693957]

18. Landon BE, et al. Improving the Management of Chronic Disease at Community Health Centers. New England Journal of Medicine. 2007; 356(9):921-934. [PubMed: 17329699]

19. Chin MH, et al. Improving and Sustaining Diabetes Care in Community Health Centers with the Health Disparities Collaboratives. Medical Care. 2007; 45(12):1135-1143. [PubMed: 18007163]

20. For example, seeBray P, et al. Feasibility and Effectiveness of System Redesign for Diabetes Care Management in Rural Areas: The Eastern North Carolina Experience. Diabetes Educator. 2005; 31(5):712-718. [PubMed: 16203855] Caruso LB, Clough-Gorr KM, Silliman RA. Improving Quality of Care for Urban Older People with Diabetes Mellitus and Cardiovascular Disease. Journal of the American Geriatrics Society. 2007; 55(10):1656-1662. [PubMed: 17714460] Also see online Appendix 2 for additional evaluations, as in Note 5.

21. Dwight-Johnson M, Ell K, Lee PJ. Can Collaborative Care Address the Needs of Low-Income Latinas with Comorbid Depression and Cancer? Results from a Randomized Pilot Study. Psychosomatics. 2005; 46(3):224-232. [PubMed: 15883143] Please also see online Appendix 2 for additional trials, as in Note 5.

22. Tsai AC, et al. A Meta-Analysis of Interventions to Improve Care for Chronic Illnesses. American Journal of Managed Care. 2005; 11(8):478-488. [PubMed: 16095434] Ouwens M, et al. Integrated Care Programmes for Chronically Ill Patients: A Review of Systematic Reviews. International Journal for Quality in Health Care. 2005; 17(2):141-146. [PubMed: 15665066] Li R, et al. Organizational Factors Affecting the Adoption of Diabetes Care Management Processes in Physician Organizations. Diabetes Care. 2004; 27(10):2312-2316. [PubMed: 15451893] others in online Appendix 2, as in Note 5.

23. Pearson, et al. Assessing the Implementation of the Chronic Care Model.

24. Parchman ML, et al. Risk of Coronary Artery Disease in Type 2 Diabetes and the Delivery of Care Consistent with the Chronic Care Model in Primary Care Settings: A STARNet Study. Medical Care. 2007; 45(12):1129-1134. [PubMed: 18007162] 
25. Fleming B, et al. The Relationship between Organizational Systems and Clinical Quality in Diabetes Care. American Journal of Managed Care. 2004; 10(12):934-944. [PubMed: 15617369]

26. Si D, et al. Assessing Health Centre Systems for Guiding Improvement in Diabetes Care. BMC Health Services Research. 2005; 5:56. [PubMed: 16117836]

27. Sperl-Hillen JM, et al. Do All Components of the Chronic Care Model Contribute Equally to Quality Improvement? Joint Commission Journal on Quality and Safety. 2004; 30(6):303-309. [PubMed: 15208979]

28. Shojania KG, et al. Effects of Quality Improvement Strategies for Type 2 Diabetes on Glycemic Control: A Meta-Regression Analysis. Journal of the American Medical Association. 2006; 296(4):427-440. [PubMed: 16868301]

29. Goetzel RZ, et al. Return on Investment in Disease Management: A Review. Health Care Financing Review. 2005; 26(4):1-19.Wagner EH, et al. Effect of Improved Glycemic Control on Health Care Costs and Utilization. Journal of the American Medical Association. 2001; 285(2):182-189. [PubMed: 11176811]

30. Gilmer TP, et al. Impact of Office Systems and Improvement Strategies on Costs of Care for Adults with Diabetes. Diabetes Care. 2006; 29(6):1242-1248. [PubMed: 16732003]

31. Huang ES, et al. The Cost Consequences of Improving Diabetes Care: The Community Health Center Experience. Joint Commission Journal on Quality and Patient Safety. 2008; 34(3):138-146. [PubMed: 18419043]

32. Huang ES, et al. The Cost-Effectiveness of Improving Diabetes Care in U.S. Federally Qualified Community Health Centers. Health Services Research. 2007; 42(6, Part 1):2174-2193. [PubMed: 17995559]

33. Mattke S, Seid M, Ma S. Evidence for the Effect of Disease Management: Is $\$ 1$ Billion a Year a Good Investment? American Journal of Managed Care. 2007; 13(12):670-676. [PubMed: 18069910] Coleman K, et al. Untangling Practice Redesign from Disease Management: How Do We Best Care for the Chronically Ill? Annual Review of Public Health (forthcoming).

34. Hung DY, et al. Rethinking Prevention in Primary Care: Applying the Chronic Care Model to Address Health Risk Behaviors. Milbank Quarterly. 2007; 85(1):69-91. [PubMed: 17319807]

35. MacColl Institute for Healthcare Innovation, RAND, and California Health Care Safety Net Institute. Integrating Chronic Care and Business Strategies in the Safety Net. Sep. 2008 Pub no. 08-0104-EFhttp://www.improvingchroniccare.org/files/CCM_Toolkit_508.pdf accessed 10 November 2008Also seeAmerican Board of Medical Specialties. IPIP: Improving Performance in Practice. http://www.abms.org/about_abms/ABMS_Research/current.aspx accessed 1 December 2008Dartmouth-Hitchcock Medical Center. Clinical Microsystems. http://dms.dartmouth.edu/cms accessed 10 November 2008 